doi: https://doi.org/10.5377/paradigma.v28i45.11739

\title{
Nivel de Desarrollo de Competencias Matemáticas en Estudiantes de Segundo Año del Profesorado en Matemáticas de la Universidad Pedagógica Nacional Francisco Morazán (UPNFM)
}

Financiado con el Fondo de Apoyo a la Investigación de la UPNFM

\section{Level of Development of Mathematical Competencies in Second Year Students of the UPNFM Mathematics Teacher's Degree Program}

\author{
Ivy Lou Green Arrechavala \\ Ledher Manuel López Urquía \\ Rosa Janeth Chambasis Cruz ${ }^{3}$
}

\begin{abstract}
Resumen
El propósito de este artículo es mostrar los resultados obtenidos a través de la investigación sobre el nivel de desarrollo de competencias matemáticas en los estudiantes de segundo año de la carrera del Profesorado en Matemáticas de la UPNFM. La metodología se desarrolló bajo un enfoque mixto basado en la complementariedad cualitativo-cuantitativo de datos. Para el diseño del modelo de evaluación de competencias matemáticas, se tomó como referencia el marco teórico del programa internacional para la Evaluación de Estudiantes (por sus siglas en inglés: Programme for International Student Assessment) PISA 2006, donde se operacionalizaron las competencias estableciendo categorías de análisis y descriptores de acuerdo con los niveles de logro de la competencia. Los resultados señalan que el mejor desempeño de los estudiantes lo alcanzan en las competencias de razonamiento y argumentación y plantear y resolver problemas. Las competencias con menor nivel de logro son la comunicación y la representación. Los niveles de competencias menos desarrollados se muestran en el desarrollo de tareas que demandan pensamiento autónomo y creatividad.
\end{abstract}

\footnotetext{
${ }^{1}$ ivy.green@upnfm.edu.hn. Doctora en Gestión del Desarrollo por la Universidad Nacional Autónoma de Honduras. https://orcid. org/0000-0001-8579-4599

${ }^{2}$ ledherlopez@gmail.com. Máster en Educación con Especialización en Formación del Profesorado por la Universidad Europea del Atlántico. España. https://orcid.org/0000-0001-8344-152X

${ }^{3}$ rschambasis@gmail.com. Profesora de Matemáticas con grado de Licenciatura por la Universidad Pedagógica Nacional Francisco Morazán. Honduras. https://orcid.org/0000-0002-3180-6694
} 
Palabras clave: competencias matemáticas, pensamiento matemático, nivel de desarrollo de competencias

\begin{abstract}
The purpose of this article is to show the results obtained through research on the level of development of mathematical competencies in second year students of the Mathematics Teacher Training Program at UPNFM. The methodology was developed under a mixed approach based on the qualitativequantitative complementarity of data. For the design of the mathematical competencies evaluation model, the theoretical framework of PISA 2006 was taken as a reference, where the competencies were operationalized by establishing categories of analysis and descriptors according to the levels of achievement of the competency. The results show that students' best performance was achieved in the competencies of reasoning and argumentation and problem posing and solving. The competencies with the lowest levels of achievement are communication and representation. The least developed levels of competencies are shown in the development of tasks that demand autonomous thinking and creativity.
\end{abstract}

Keywords: mathematical competencies, mathematical thinking, competency development level

\title{
Introducción
}

La sociedad basada en el conocimiento, la internacionalización y la dinámica de las últimas décadas han producido grandes cambios en la sociedad. Este nuevo contexto ha trascendido a la educación y sus demandas obligan a crear un nuevo enfoque, más allá de los contenidos se requiere procesos educativos que desarrollen competencias, que promueva personas con capacidades para integrarse en el mundo actual y para seguir aprendiendo y fortaleciendo sus saberes (ser, conocer, hacer y convivir) a lo largo de la vida.

En el caso de Honduras, es hacia el año 2006 cuando la Universidad Pedagógica Nacional Francisco Morazán (UPNFM), inicia un proceso de reforma curricular con el fin de superar las debilidades detectadas como resultado de la autoevaluación realizada entre el 2000 y 2003. Después de revisar varias tipologías y conceptualizaciones sobre reformas curriculares actuales, se adscribe al Proyecto Tuning América Latina ${ }^{4}$ el cual propone un sistema de competencias como elementos articuladores para diseñar planes de estudio de las titulaciones de educación

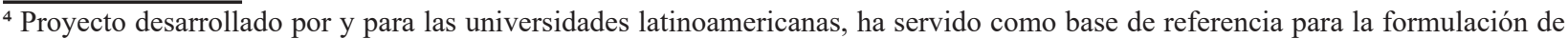
competencias trasversales y específicas que se tomaron en cuenta en los nuevos planes desarrollados por la UPNFM, después de consultar acerca de las competencias deseables a empleadores, docentes, graduados y estudiantes de la UPNFM
} 
superior. El proceso de reforma curricular de la UPNFM se concreta en el año 2008, al establecer para todas las carreras una propuesta curricular basada en competencias, incluyendo la carrera del Profesorado en Matemática.

Conscientes que un cambio curricular implica, como lo señala Barrales (2012), "no sólo un cambio en la metodología de trabajo sino también en la forma en que el docente percibe la realidad" (p.32) y que en esto la tradición tiene mucho peso, después de cinco años de vigencia del curriculum reformado, se genera el interés en esta investigación cuyo objetivo es describir el nivel de desarrollo que muestran los estudiantes del segundo año de la carrera de Matemáticas de la UPNFM de la modalidad presencial, en las competencias matemáticas seleccionadas.

Como antecedente teórico de esta investigación se considera esencial la propuesta de Niss (2002), Niss y Hojgaard (2011) y la OCDE(2006) en su propuesta para el Marco Teórico de PISA, para quienes las competencias matemáticas incluyen: la disposición de pensar matemáticamente, el placer y la seguridad en el desarrollo de actividades intelectuales que implican el razonamiento matemático, la capacidad para argumentar y comunicar el pensamiento matemático en forma oral y escrita y la capacidad para comprender y solucionar problemas matemáticos.

También se toma como precedente diferentes proyectos que definieron competencias matemáticas deseables tales como MAT747 de Abrantes (2001) en Portugal, el proyecto KOM de Niss (2002) en Dinamarca, el proyecto PISA de la OECD (2006), Principios y Estándares para la Educación Matemática en Estados Unidos (NCTM, 2000) y otros orientados a la evaluación como Trends in International Mathematics and Science Study (TIMSS), el Instituto de Fomento de la Educación (ICFES) y las pruebas SABER en Colombia, el Programa de Evaluación PanCanadiense (PCAP) de Canadá, los cuales de manera general coinciden en que las competencias matemáticas son: razonar y argumentar, comunicar, modelar, plantear y resolver problemas, representar, utilizar lenguaje simbólico, formal y técnico y de las operaciones y uso de herramientas y recursos que facilitan la actividad matemática, en contextos, modos y situaciones en que son presentadas.

Este estudio se delimita en el abordaje de cuatro competencias matemáticas: planteamiento y solución de problemas, razonamiento y argumentación, comunicación y representación. Como valor agregado se aporta un modelo para la evaluación de dichas competencias. 


\section{Discusión Teórica}

Para atender las demandas a la educación, creadas por una sociedad del conocimiento, un mundo globalizado cargado de incertidumbres surge el enfoque basado en competencias. Investigadores como Gonzáles y Gonzáles (2008) focalizan las competencias en el ámbito laboral. Tobón (2008) ofrece un análisis que retoma datos históricos sobre la evolución del concepto de competencias hasta su impacto en educación, enfatizando en la educación superior y un enfoque socioformativo, en cuyo marco las competencias se abordan como actuaciones integrales para resolver problemas de la vida con idoneidad, ética y mejora continua.

La necesidad de adoptar una postura referente al concepto de competencias se debe a que el término es polisémico y que además se ha ido reconceptualizando en atención a las demandas de la formación humana y ciudadana. De ahí que el concepto de competencia, adoptado para esta investigación se fundamenta en la visión propuesta por Delors (1996) donde los pilares de la educación apuntan hacia una inclusión de componentes cognitivos, como son los conocimientos y habilidades y también componentes afectivos y axiológicos y a la propuesta de saberes integrados de Tobón (2010) y D’Ámore et al. (2008), como se muestra en la Figura 1.

\section{Figura 1}

Competencia como integración de saberes

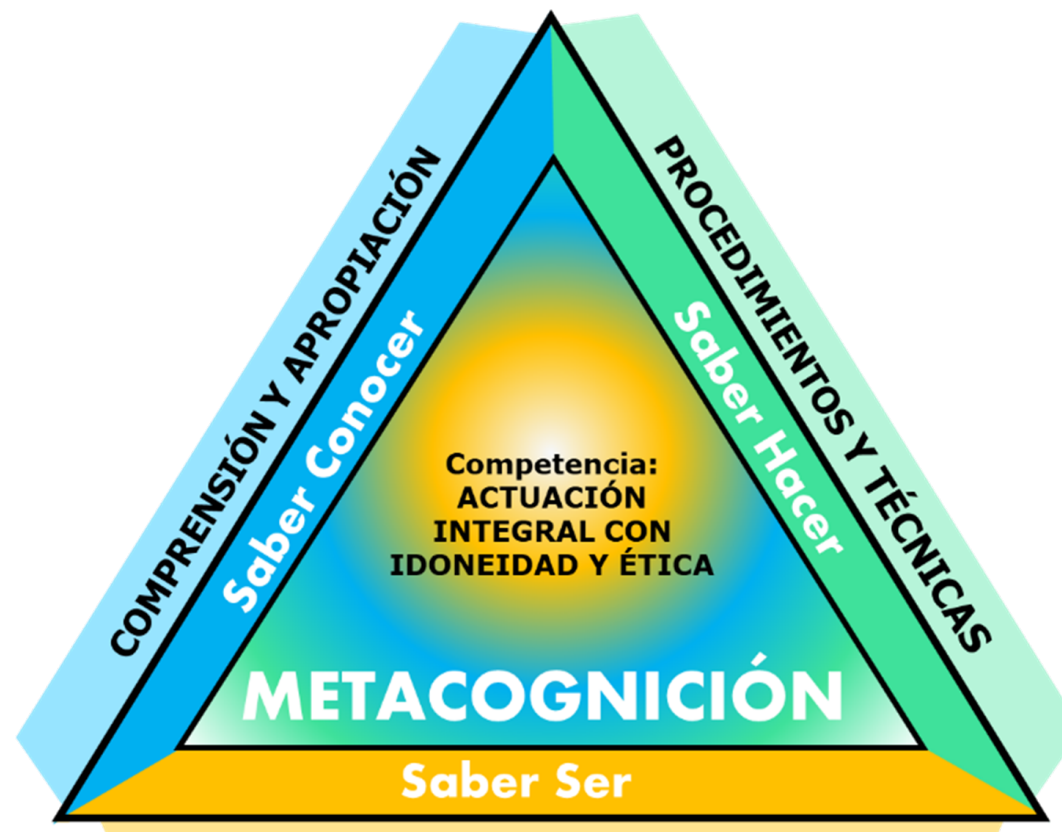

ACTITUDES Y VALORES

Nota. Elaboración de los autores adaptado de Tobón (2010) y D’Ámore et al. (2008) 
La formación basada en competencia implica un cambio de paradigma frente a la educación tradicional. Exige que el alumno sepa leer, interpretar y actuar en situaciones próximas a la realidad, considerando la complejidad que envuelve la situación presentada. El ejercicio de la competencia implica la movilización integral de tres saberes, que buscan desarrollar la metacognición, a diferencia de las posturas tradicionales de saberes desarticulados y descontextualizados las competencias se muestran en la acción y la acción debe tener un contexto.

\section{Aproximaciones Conceptuales a la Noción de Competencias Matemáticas}

En la literatura especializada se encuentra una caracterización de competencias clasificándolas en competencias genéricas, específicas profesionales y específicas disciplinares. Las competencias genéricas son aquellas comunes a todas las personas, las competencias específicas son aquellas que abordan explícitamente habilidades, conocimientos y actitudes que caracterizan a un profesional de un área específica. En ese sentido, en el caso de la carrera de profesorado en matemática, las competencias específicas disciplinares hacen referencia a las competencias matemáticas.

Al respecto, la investigación documental lleva a afirmar que en Honduras no existen trabajos investigativos previos referidos al tema en cuestión o si existen no se encuentran publicados, por lo cual se desconoce el nivel de desarrollo de este campo de conocimiento en nuestro contexto. En contraste, en el ámbito internacional se han planteado proyectos similares que profundizan sobre el tema de competencias matemáticas.

El análisis documental de los proyectos e informes de investigación que se mencionan en la introducción, así como el Plan de Estudio de la Carrera de Matemáticas en el Grado de Licenciatura de la UPNFM (2008), permite dos cosas, la primera, asumir como concepto de competencia matemática la postura del Programa PISA(OCDE, 2006), que concibe a la competencia matemática como:

La capacidad que tiene un individuo de identificar y comprender el papel que desempeñan las matemáticas en el mundo, emitir juicios bien fundados y utilizar e implicarse en las matemáticas de una manera que satisfaga sus necesidades vitales como un ciudadano constructivo, comprometido y reflexivo (p.13)

Y la segunda, seleccionar las competencias matemáticas para esta investigación. En este sentido, la investigación se limita a cuatro competencias matemáticas: (1) razonamiento y argumentación; (2) plantear y resolver problemas, (3) comunicación y (4) representación (matemática). En este 
estudio, a partir de la propuesta de Niss y Hojgaard (2011), se definió el dominio de cada competencia matemática, de la siguiente manera:

El dominio de la competencia comunicación se muestra si la persona:

comunica, expresa y presenta conocimiento, razonamientos matemáticos o conclusiones con claridad, precisión y rigurosidad, utilizando un lenguaje adecuado tanto verbal, oral o escrito, o el no verbal, tomando en consideración la audiencia a la que se dirige, los diferentes sentidos e intenciones de la comunicación y los recursos tecnológicos.

La persona que domina la competencia de representación:

construye y manipula imágenes mentales de objetos e ideas matemáticas, usando papel y lápiz, software matemático, modelos físicos u otros recursos; sus transformaciones y sus diversas traducciones, de manera correcta realizando conexiones matemáticas, a fin de resolver problemas o situaciones de la vida real con éxito.

El dominio de la competencia de plantear y resolver problemas implica que la persona: resuelve, traduce y demuestra problemas matemáticos que le permitan dar solución a problemas contextualizados en la vida cotidiana, pretendiendo desarrollar la capacidad para proyección social, la movilización de los saberes necesarios para el desempeño profesional exitoso y entre otras cosas, una actitud crítica y propositiva en la búsqueda y solución de los problemas, con una visión de educación permanente y con conciencia de la responsabilidad profesional.

El dominio de la competencia de razonamiento y argumentación se manifiesta cuando la persona: distingue entre distintos tipos de asertos (definiciones, teoremas, conjeturas, hipótesis, ejemplos, afirmaciones condicionales); comprende y sabe manejar el alcance y los límites de los conceptos matemáticos que hagan al caso; entiende en qué consisten las pruebas matemáticas y qué la diferencia de otro tipo de razonamiento matemático; sigue y evalúa cadenas de argumentaciones matemáticas de distintos tipos; tiene un sentido heurístico, así como crea y expresa argumentaciones matemáticas.

En conclusión, para efectos de esta investigación el dominio de competencias se observa especificando los desempeños que el estudiante muestra en el desarrollo de la tarea que corresponde a determinada competencia. Se establecen distintos niveles de logro o dominio de la competencia mediante los indicadores de logro y descriptores según la complejidad de cada tarea a resolver. 
El nivel de desarrollo de las competencias se valora de acuerdo con los aprendizajes logrados, esto se entiende como lo que "un estudiante sabe, comprende y/o es capaz de hacer al término de un periodo de aprendizaje" (CSUCA 2013, p. 11).

\section{Métodos y Materiales}

En este estudio se utilizó un enfoque mixto, como un proceso que recolecta, analiza y vincula datos cuantitativos y cualitativos en un mismo estudio. En este caso, la complementariedad se da a través del estudio de casos mediante variables cualitativas, donde posteriormente para efectos de la descripción de resultados, se hace un estudio cuantitativo sobre los tipos de respuestas obtenidas por los participantes. El alcance del estudio es descriptivo - transversal. La investigación pretende describir, reflexionar y caracterizar con rigor, los hallazgos encontrados, en un tiempo y espacio específico en relación con el objeto de estudio en este caso el nivel de desarrollo de las competencias matemáticas que muestran los participantes.

\section{Población y Muestra}

La población de la investigación está conformada por todos los estudiantes que cursan el segundo año de la carrera de matemáticas en el sistema presencial, en la sede de Tegucigalpa.

Mientras que la muestra del proyecto se determinó a través de un muestreo no probabilístico dirigido, se conformó con 15 sujetos que reunían dos características principales (1) estar cursando los espacios pedagógicos de Geometría II o Cálculo III, en consideración a su ubicación dentro del pénsum académico según el diseño curricular del plan de estudios (segundo año) y no referida a la fecha de ingreso del estudiante y (2) tener un índice académico mayor de 70\%, este criterio va en consonancia con el objetivo de la investigación.

\section{Procedimiento de la Investigación}

Dada la extensión de la investigación y los distintos aspectos que abarcaba se tomó a bien dividir el proceso de ejecución en tres etapas.

Primera Etapa: Operacionalización de competencias.

Segunda Etapa: Construcción de instrumentos, validación y aplicación de los mismos.

Tercera Etapa: Procesamiento de los datos obtenidos.

\section{Primera Etapa: Operacionalización de Competencias}

Un paso importante en el desarrollo de la investigación fue la operacionalización de las variables en este caso, las competencias matemáticas. Este proceso obliga a realizar una definición conceptual 
para eliminar ambigüedades y así darle sentido concreto dentro de la investigación, luego en función de ello se procede a realizar la definición operacional usando factores estrictamente evaluables, visibles, a los que se les llama indicadores de logro, que a su vez permitan realizar la valoración del nivel de logro de la competencia de acuerdo con un desempeño mostrado.

Esta actividad se planteó y desarrolló en la primera etapa del proyecto, la cual puede verse en Green, Chambasis, López, Valladares, Díaz, Martínez y Molina (2014) donde se desarrolla una aproximación a la operacionalización de competencias matemáticas. A continuación, la Figura 2 sintetiza el proceso seguido para la operacionalización de competencias y una descripción mínima de cada elemento.

\section{Figura 2}

Proceso de operacionalización de competencias

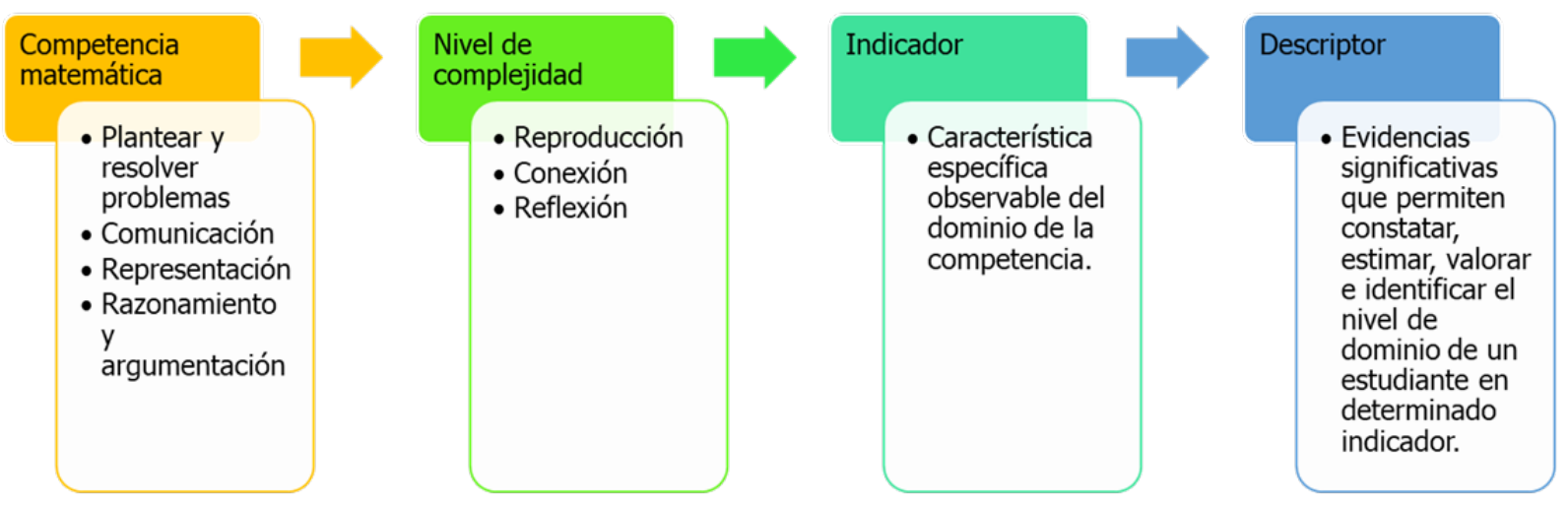

Nota. Fuente: elaboración de los autores

Siguiendo el procedimiento descrito y al no contar con una experiencia nacional en cuanto a la operacionalización de competencias, esta operacionalización se perfilaba como un elemento importante para la investigación y para la evaluación de competencias en futuras experiencias. En la descripción de resultados se presenta un ejemplo de esta operacionalización en consideración a un ítem en específico.

\section{Segunda Etapa: Elaboración de Prueba de Desempeño}

Diseñado el modelo de evaluación de competencias matemáticas, se procedió a la realización de la segunda etapa del proyecto que consistió en la elaboración de la prueba de desempeño, la elaboración del cuestionario y su aplicación. El análisis de los niveles de competencia alcanzados por los estudiantes corresponde al desempeño mostrado en el desarrollo de la prueba según los descriptores preparados para tal fin. La construcción y selección de ítems se hizo tomando en cuenta 
las dimensiones que caracterizan y son requeridas para evaluar las competencias matemáticas como se presenta en la Figura a continuación.

\section{Figura 3}

Dimensiones que caracterizan a las competencias matemáticas

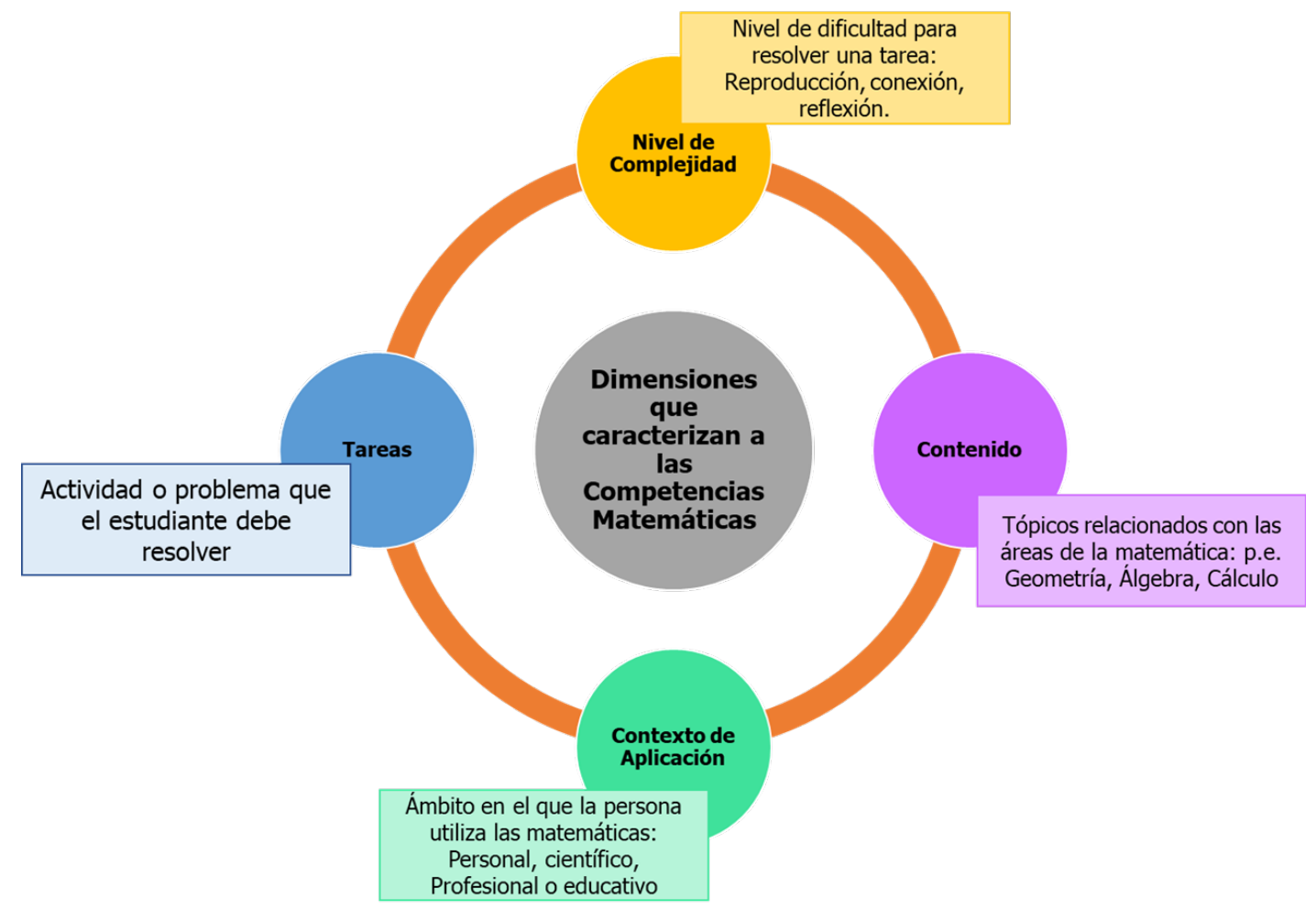

Nota. Fuente: elaboración de los autores

Atendiendo las dimensiones requeridas para evaluar competencias, la cvonfiguración de la prueba de desempeño se delimita de acuerdo con las siguientes características:

- Competencias matemáticas: razonamiento y argumentación, representación, comunicación y planteamiento y resolución de problemas.

- Contenido: Álgebra, Geometría y Cálculo, tomando en cuenta las clases matemáticas cursadas hasta el 2do año de carrera.

- Contexto de aplicación: laboral y científico.

- Tarea o situación: magnitudes, derivada, vectores en $\mathrm{R}^{2}$, expresiones algebraicas, ángulos entre paralelas, objetos en el plano y espacio, función lineal.

- Nivel de complejidad: Dado que las tareas propuestas a los estudiantes deben plantear diferentes tipos y niveles de demandas cognitivas, esta variable se refiere al nivel de dificultad de la situación problema planteado (PISA, 2006, p. 112). Se plantean los siguientes niveles de complejidad: 
»Reproducción: en el nivel de reproducción se engloban aquellos ejercicios que son familiares y que exigen básicamente la reiteración de los conocimientos practicados, como son las representaciones de hechos y problemas comunes, recuerdo de objetos y propiedades matemáticas familiares, reconocimiento de equivalencias, utilización de procesos rutinarios, aplicación de algoritmos, manejo de expresiones con símbolos y fórmulas familiares, o la realización de operaciones sencillas.

»Conexión: el nivel de conexiones permite resolver problemas que no son simplemente rutinarios, pero que están situados en contextos familiares o cercanos. Plantean mayores exigencias para su interpretación y requieren establecer relaciones entre saberes de distintas áreas de la matemática y distintas formas de representar una misma situación, o bien enlazar diferentes aspectos con el fin de alcanzar una solución.

»Reflexión: las tareas en este nivel de complejidad movilizan competencias que requieren cierta comprensión y reflexión por parte del alumno, creatividad para identificar conceptos o enlazar conocimientos de distintas procedencias. Las tareas de este nivel requieren competencias más complejas, implican un mayor número de elementos cognitivos, exigen generalización y explicación o justificación de los resultados.

Finalmente, el instrumento quedó integrado por 12 ítems, 10 de respuesta abierta y dos de selección, los que como se puede ver en la Tabla 1 incluyen diferentes áreas de la matemática, plantean demandas o habilidades cognitivas diferentes para su resolución según los distintos niveles de complejidad de la tarea. La Tabla 1 presenta el resumen de la caracterización de los ítems incluidos en la prueba de desempeño.

\section{Tabla 1}

Resumen de las dimensiones utilizadas en la elaboración de items.

\begin{tabular}{ccccc}
\hline $\begin{array}{c}\text { Número } \\
\text { de Ítem }\end{array}$ & Contenido & Tarea o situación Problema & Contexto & $\begin{array}{c}\text { Nivel de } \\
\text { complejidad }\end{array}$ \\
\hline 1 & Geometría & Estimación de magnitudes. & Científico & Reproducción \\
2 & Geometría & Estimación de magnitudes. & Científico & Reproducción \\
3 & Cálculo $^{5}$ & Aplicaciones de la derivada & Científico & Reproducción \\
4 & Álgebra & $\begin{array}{l}\text { Traducción de enunciados a } \\
\text { expresiones algebraicas. }\end{array}$ & Científico & Reproducción \\
\hline \hline
\end{tabular}

${ }_{5}^{5}$ Percibido como una prolongación del área matemática de algebra, debido a que dicho contenido se aborda en el plan de la carrera a nivel de segundo año. 


\begin{tabular}{|c|c|c|c|c|}
\hline $\begin{array}{l}\text { Número } \\
\text { de Ítem }\end{array}$ & Contenido & Tarea o situación Problema & Contexto & $\begin{array}{c}\text { Nivel de } \\
\text { complejidad }\end{array}$ \\
\hline 5 & Geometría & $\begin{array}{l}\text { Aplicación del Teorema de } \\
\text { Pitágoras }\end{array}$ & Científico & Reproducción \\
\hline 6 & Álgebra & Aplicación de vectores en $\mathrm{R}^{2}$ & Laboral & Reflexión \\
\hline 7 & Geometría & Ángulo entre rectas paralelas & Científico & Reflexión \\
\hline 8 & Geometría & $\begin{array}{l}\text { Representación de objetos en el } \\
\text { plano y la relación entre ellos }\end{array}$ & Laboral & Reflexión \\
\hline 9 & Geometría & $\begin{array}{l}\text { Representación de objetos en el } \\
\text { espacio y la relación entre ellos }\end{array}$ & Laboral & Conexión \\
\hline 10 & Álgebra & $\begin{array}{l}\text { Aplicación de sistema de ecuaciones } \\
\text { lineales en una variable }\end{array}$ & Científico & Conexión \\
\hline 11 & Álgebra & $\begin{array}{l}\text { Representación gráfica de una } \\
\text { Función Lineal }\end{array}$ & Laboral & Conexión \\
\hline 12 & Álgebra & $\begin{array}{l}\text { Lectura e interpretación del } \\
\text { comportamiento de una función lineal }\end{array}$ & Laboral & Conexión \\
\hline
\end{tabular}

Nota. Fuente: Elaboración de los autores.

El instrumento se validó mediante criterios de expertos y prueba piloto. Como expertos participaron 10 docentes del departamento de Ciencias Matemáticas y el pilotaje se ejecutó con 25 estudiantes de la carrera de matemáticas que cursaban el tercer año académico en la UPNFM. Los resultados de este proceso, al finalizar la validación, mostraron alto índice de validez y confiabilidad.

\section{Tercera Etapa: Aplicación y Análisis de Datos}

La tercera y última etapa contempla el análisis de los datos obtenidos a través de la prueba aplicada. El análisis de los datos se orienta a establecer los niveles de desarrollo mostrado por los estudiantes en las competencias matemáticas incluidas en este estudio. Según el modelo propuesto, el nivel de dominio de la competencia se describe por las etapas sucesivas en el desarrollo de la competencia, especificando los desempeños que el estudiante debe mostrar en el desarrollo de la tarea. Estas se relacionan con los descriptores propios de cada nivel de logro y de acuerdo con el nivel de complejidad de la tarea propuesta en relación con las competencias matemáticas. 
Para el análisis de cada ítem se prepararon las rúbricas correspondientes siguiendo los indicadores y descriptores propuestos en la operacionalización de la competencia. Siguiendo los criterios establecidos, primeramente, se analizó el trabajo realizado por los estudiantes de manera cualitativa, se categorizaron los casos de acuerdo con los rasgos mostrados en el proceso de solución de las situaciones o tareas planteadas.

Para establecer el nivel de desarrollo de las competencias, se identificaron cuatro categorías: nulo, inicial, avanzado y experto, para lo cual se asignó un código según el nivel alcanzado en el desarrollo de cada ítem de la prueba de desempeño, tomando como elementos de comparación y decisión los descriptores de cada nivel de complejidad de la tarea. Los niveles de logro o desarrollo de la competencia se identifican de la siguiente manera:

- Nulo: entendiéndose como un nivel en donde el estudiante no resuelve el ejercicio o lo que muestra no son evidencias suficientes del dominio de la competencia.

- Inicial: el estudiante demuestra conocimientos, habilidades y destrezas mínimas del dominio de la competencia. El estudiante en este nivel tiene un desempeño mínimo aceptable de la competencia evaluada.

- Avanzado: El estudiante muestra los conocimientos, habilidades y destrezas necesarios en el dominio de la competencia. El estudiante en este nivel demuestra un desempeño aceptable de la competencia investigada.

- Experto: El estudiante demuestra alto dominio de conocimientos, habilidades y destrezas relacionados con la competencia. El estudiante en este nivel demuestra un desempeño superior en el uso de la competencia evaluada.

- Debido a los resultados observados en la prueba de desempeño se tuvo que agregar otra categoría a la que se denominó Inferior (sólo para conexión y reflexión): Entendiéndose como un nivel donde las evidencias del trabajo realizado por el estudiante no solo no alcanzan los requerimientos mínimos de la competencia sino que son incoherentes.

Una vez analizados los cuestionarios de cada estudiante, se contabilizan las frecuencias, en cada nivel de logro y cada nivel de complejidad y se contrastaron sus diferencias y similitudes. Para describir el nivel general alcanzado en cada competencia, se realizó un promedio de las frecuencias de cada nivel de desarrollo por competencia. En un segundo momento se analizaron los niveles de complejidad según los niveles de desarrollo para finalmente poder realizar gráficos comparativos. 


\section{Proceso de Análisis de Datos}

Como ilustración del tratamiento que siguió para el análisis de los niveles de desarrollo de las competencias se presenta un ejemplo. A continuación, se presentan los elementos del proceso que permitieron el análisis del ítem 6 de la prueba de desempeño.

Elemento 1. La Tabla 2 presenta la descripción del ítem. Cada problema tiene su número, el contenido donde se encuentra, la tarea específica, el contexto y un nivel de complejidad.

\section{Tabla 2}

Descripción del Ítem No. 6

\begin{tabular}{|c|c|c|}
\hline \\
\hline No. de ítem & 6 & $\begin{array}{l}\text { El tío Pedro era un hombre que le gustaban las formas, un día } \\
\text { le ofrecieron comprar un campo y él acepto con la condición } \\
\text { que le dejaran comprar solo lo que estuviera dentro de un }\end{array}$ \\
\hline Contenido & Algebra & \\
\hline Tarea & $\begin{array}{l}\text { Aplicaciones de } \\
\text { direcciones en } \mathrm{R}^{2}\end{array}$ & \\
\hline Contexto & Laboral & $\begin{array}{l}\text { que representa el tercer vértice y termina dirigiéndose con la } \\
\text { dirección del rumbo } \mathrm{C} \text { al punto de inicio o sea el primer vértice. } \\
\text { Determine si el siguiente enunciado es verdadero o falso: }\end{array}$ \\
\hline $\begin{array}{c}\text { Nivel de } \\
\text { complejidad }\end{array}$ & Reflexiones & \\
\hline
\end{tabular}

Elemento 2. La Tabla 3 presenta la rúbrica que especifica los niveles de logro de la competencia, esta se desglosa de la operacionalización previamente elaborada. Se convierte en una rúbrica operativa cuando incorporamos evaluación en el ítem, el cual responde al indicador y descriptor. Esto nos permite indicar si el estudiante realiza o no dicha acción y a partir de ello inferir el nivel de desarrollo o logro en el dominio de la competencia. De esta manera en forma descendente, cada descriptor corresponde a un nivel de desarrollo, es decir, inicial, avanzado y experto. 


\section{Tabla 3}

Rubrica basada en la operacionalización para evaluar el Ítem No. 6

\begin{tabular}{|c|c|c|c|}
\hline Competencia & $\begin{array}{c}\text { Indicador de } \\
\text { logro } \\
\end{array}$ & Descriptor & Evaluación en el ítem \\
\hline \multirow{3}{*}{$\begin{array}{c}1 . \\
\text { Comunicación }\end{array}$} & \multirow{3}{*}{$\begin{array}{l}\text { Comprende } \\
\text { y expresa } \\
\text { oralmente y } \\
\text { por escrito } \\
\text { cuestiones } \\
\text { matemáticas } \\
\text { en situaciones } \\
\text { no familiares } \\
\text { explicando } \\
\text { cálculos, } \\
\text { resultados } \\
\text { desarrollados } \\
\text { en la solución } \\
\text { de problemas } \\
\text { que implican } \\
\text { relaciones } \\
\text { complejas entre } \\
\text { ellas relaciones } \\
\text { lógicas }\end{array}$} & $\begin{array}{l}\text { Interpreta la situación expuesta, opera } \\
\text { matemáticamente pero no comunica } \\
\text { ni explica cálculos y resultados } \\
\text { desarrollados en la solución de } \\
\text { problemas que implican relaciones } \\
\text { complejas entre ellas relaciones } \\
\text { lógicas. }\end{array}$ & $\begin{array}{l}\text { Inicial. Interpreta la situación expuesta de } \\
\text { la actividad del topógrafo y de los rumbos } \\
\text { que llevan a los vértices de un triángulo } \\
\text { y cuando opera matemáticamente no } \\
\text { comunica ni explica sus cálculos ni } \\
\text { resultados obtenidos en la resolución del } \\
\text { problema que implica relaciones lógicas } \\
\text { entre distancias recorridas con longitudes } \\
\text { de un triángulo isósceles en el plano } \\
\text { cartesiano. }\end{array}$ \\
\hline & & $\begin{array}{l}\text { Interpreta la situación expuesta, opera } \\
\text { matemáticamente y comunica sus ideas } \\
\text { matemáticas, nombres y propiedades } \\
\text { en procedimientos y algoritmos } \\
\text { habituales, pero no explica sus } \\
\text { cálculos, y resultados desarrollados en } \\
\text { la solución de problemas que implican } \\
\text { relaciones complejas entre ellas } \\
\text { relaciones lógicas. }\end{array}$ & $\begin{array}{l}\text { Avanzado. Interpreta la situación } \\
\text { expuesta de la actividad del topógrafo } \\
\text { y de los rumbos que llevan a los } \\
\text { vértices de un triángulo y cuando opera } \\
\text { matemáticamente comunica sus ideas } \\
\text { matemáticas con propiedades utilizadas, } \\
\text { pero no explica sus cálculos ni resultados } \\
\text { obtenidos que implican relaciones lógicas } \\
\text { entre distancias recorridas con longitudes } \\
\text { de los lados de un triángulo isósceles en el } \\
\text { plano cartesiano }\end{array}$ \\
\hline & & $\begin{array}{l}\text { Interpreta la situación expuesta, opera } \\
\text { matemáticamente y comunica sus ideas } \\
\text { matemáticas, nombres y propiedades en } \\
\text { procedimientos y algoritmos habituales } \\
\text { además explica sus cálculos, y } \\
\text { resultados desarrollados en la solución } \\
\text { de problemas que implican relaciones } \\
\text { complejas entre ellas relaciones } \\
\text { lógicas. }\end{array}$ & $\begin{array}{l}\text { Experto. Interpreta la situación expuesta } \\
\text { de la actividad del topógrafo y de } \\
\text { los rumbos que llevan a los vértices } \\
\text { de un triángulo y cuando opera } \\
\text { matemáticamente comunica sus ideas } \\
\text { matemáticas con propiedades utilizadas, } \\
\text { explica sus cálculos y resultados obtenidos } \\
\text { que implica relaciones lógicas entre } \\
\text { distancias recorridas con longitudes de un } \\
\text { triángulo isósceles en el plano cartesiano }\end{array}$ \\
\hline \multirow{3}{*}{$\begin{array}{c}2 . \\
\text { Representación }\end{array}$} & \multirow{3}{*}{$\begin{array}{l}\text { Descodifica, } \\
\text { codifica e } \\
\text { interpreta } \\
\text { formas de } \\
\text { representación } \\
\text { de conceptos } \\
\text { matemáticos, } \\
\text { en situaciones } \\
\text { no familiares, } \\
\text { selecciona } \\
\text { y cambia } \\
\text { representaciones } \\
\text { de manera } \\
\text { creativa } \\
\end{array}$} & $\begin{array}{l}\text { Descodifica una representación, pero } \\
\text { no logra interpretarla adecuadamente } \\
\text { utilizando un cambio de representación } \\
\text { incorrecto }\end{array}$ & $\begin{array}{l}\text { Inicial. Representa incorrectamente } \\
\text { las direcciones en } \mathrm{R}^{2} \text { y las distancias } \\
\text { recorridas como longitudes de un } \\
\text { triángulo isósceles asumiendo información } \\
\text { incorrecta o no proporcionada. }\end{array}$ \\
\hline & & $\begin{array}{l}\text { Descodifica una representación } \\
\text { en situaciones no familiares y la } \\
\text { interpreta de manera correcta, pero el } \\
\text { registro de representación utilizado es } \\
\text { parcialmente correcto. }\end{array}$ & $\begin{array}{l}\text { Avanzado. Representa de forma parcial } \\
\text { las direcciones en } \mathrm{R}^{2} \mathrm{y} \text { las distancias } \\
\text { recorridas con longitudes de un triángulo } \\
\text { isósceles. }\end{array}$ \\
\hline & & $\begin{array}{l}\text { Descodifica una representación en } \\
\text { situaciones no familiares la interpreta } \\
\text { de manera correcta reflexionando sobre } \\
\text { el registro de representación utilizado }\end{array}$ & $\begin{array}{l}\text { Experto. Representa correctamente los } \\
\text { rumbos en } \mathrm{R}^{2} \text { y distancias recorridas con } \\
\text { longitudes de un triángulo isósceles }\end{array}$ \\
\hline
\end{tabular}




\section{Continúa Tabla 3}

\begin{tabular}{|c|c|c|c|}
\hline Competencia & $\begin{array}{c}\text { Indicador de } \\
\text { logro }\end{array}$ & Descriptor & Evaluación en el ítem \\
\hline \multirow{3}{*}{$\begin{array}{l}\text { 3. Plantear } \\
\text { y Resolver } \\
\text { problemas }\end{array}$} & \multirow{3}{*}{$\begin{array}{l}\text { Expone y } \\
\text { formula } \\
\text { problemas } \\
\text { mediante la } \\
\text { utilización de } \\
\text { procedimientos } \\
\text { y aplicación } \\
\text { estándar pero } \\
\text { también de } \\
\text { procedimientos } \\
\text { más originales } \\
\text { que implican } \\
\text { establecer } \\
\text { conexiones } \\
\text { entre distintas } \\
\text { áreas } \\
\text { matemáticas } \\
\text { y distintas } \\
\text { formas de } \\
\text { representación. } \\
\text { También } \\
\text { conlleva } \\
\text { reflexionar } \\
\text { sobre las } \\
\text { estrategias } \\
\text { de solución y } \\
\text { resultados en } \\
\text { situaciones no } \\
\text { familiares }\end{array}$} & $\begin{array}{l}\text { Interpreta adecuadamente el } \\
\text { enunciado, aplicando procedimientos } \\
\text { o estrategias matemáticas incoherentes } \\
\text { con los datos del problema. }\end{array}$ & $\begin{array}{l}\text { Inicial. Interpreta adecuadamente el } \\
\text { enunciado de la actividad del topógrafo } \\
\text { y la relación con un triángulo isósceles, } \\
\text { pero el procedimiento de resolución } \\
\text { es incorrecto o incoherente con las } \\
\text { direcciones dadas en el problema }\end{array}$ \\
\hline & & $\begin{array}{l}\text { Interpreta adecuadamente el } \\
\text { enunciado, aplicando procedimientos } \\
\text { matemáticos parcialmente correctos; } \\
\text { no reflexiona sobre los procedimientos } \\
\text { aplicados y resultados obtenidos. }\end{array}$ & $\begin{array}{l}\text { Avanzado. Interpreta adecuadamente el } \\
\text { enunciado de la actividad del topógrafo } \\
\text { y la relación con un triángulo isósceles, } \\
\text { pero el procedimiento de resolución no es } \\
\text { adecuado ni reflexiona sobre su estrategia } \\
\text { de resolución de los rumbos dados en } \mathrm{R}^{2} \\
\text { con las longitudes y distancias. }\end{array}$ \\
\hline & & $\begin{array}{l}\text { Interpreta adecuadamente el } \\
\text { enunciado, el procedimiento } \\
\text { matemático es pertinente llega a } \\
\text { la respuesta correcta y reflexiona } \\
\text { sobre los procedimientos aplicados y } \\
\text { resultados obtenidos discriminando } \\
\text { entre las mejores alternativas de } \\
\text { solución. }\end{array}$ & $\begin{array}{l}\text { Experto. Interpreta adecuadamente el } \\
\text { enunciado de la actividad del topógrafo y } \\
\text { la relación con un triángulo isósceles, el } \\
\text { procedimiento de resolución es adecuado } \\
\text { y su respuesta es correcta reflexionando } \\
\text { sobre su estrategia de resolución de los } \\
\text { rumbos dados en } \mathrm{R}^{2} \text { con las longitudes y } \\
\text { distancias }\end{array}$ \\
\hline
\end{tabular}

Argumenta utilizando propiedades

Mantiene una y relaciones entre algunos objetos actitud activa y matemáticos en situaciones complejas reflexiva para argumentar

4. y evaluar Razonamiento A encadenamientos matemáticos en situaciones no familiares usando la heurística y la intuición generaliza o nuevas, pero es incorrecta.

Argumenta y evalúa utilizando propiedades y relaciones entre algunos objetos matemáticos en situaciones complejas o nuevas, pero es incompleta.

Argumenta y evalúa utilizando propiedades y relaciones entre objetos matemáticos en situaciones complejas o nuevas, justificando correctamente / generaliza la solución
Inicial. Argumenta utilizando propiedades y relaciones entre los rumbos en $\mathrm{R}^{2}$, longitudes y distancias, pero su justificación es incorrecta.

Avanzado. Argumenta y evalúa utilizando propiedades y relaciones entre los rumbos en $\mathrm{R}^{2}$, longitudes y distancias, pero su justificación es incompleta.

Experto. Argumenta y evalúa utilizando propiedades y relaciones entre los rumbos en $\mathrm{R}^{2}$, longitudes y distancias, justificando correctamente / generaliza la solución 
Elemento 3. La Figura 4 ofrece, a manera de ejemplo una resolución del trabajo del estudiante, se revisa conforme a la rúbrica y a partir de lo observado y para cada competencia se posiciona el trabajo del estudiante en un nivel de logro determinado según la Tabla 3.

\section{Figura 4}

Resolución del Ítem 6 por el estudiante No. 2

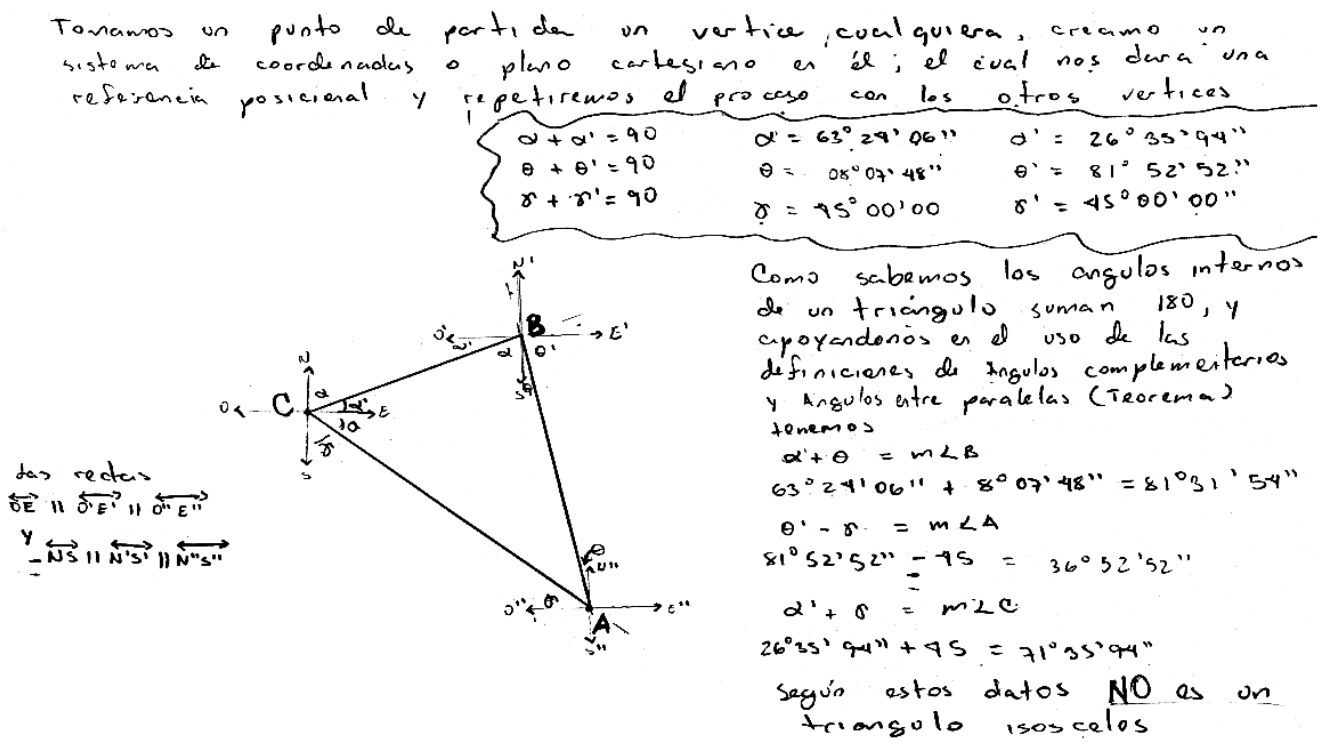

Elemento 4. Valoración del ítem de acuerdo con las evidencias de desempeño que muestra el participante.

\section{Tabla 4}

Valoración del Ítem No. 6 para el estudiante No. 2

\begin{tabular}{llc}
\hline Competencia & \multicolumn{1}{c}{ Evaluación de evidencias } & $\begin{array}{c}\text { Nivel en el que se } \\
\text { encuentra }\end{array}$ \\
\hline \multirow{3}{*}{ Comunicación } & $\begin{array}{l}\text { Interpreta el enunciado, encuentra relaciones que le permiten } \\
\text { resolver el problema explicando con propiedades matemáticas } \\
\text { su procedimiento de solución. Además, explica el resultado } \\
\text { obtenido verificando si en su solución los ángulos encontrados } \\
\end{array}$ & Nivel Experto \\
& son iguales y corresponden a un triángulo isósceles. & \\
& $\begin{array}{l}\text { Descodifica la representación de los rumbos que toma el } \\
\text { topógrafo e interpreta correctamente que los rumbos trazados } \\
\text { Representación }\end{array}$ & \\
& $\begin{array}{l}\text { deberían formar un triángulo isósceles. Su representación es } \\
\text { correcta y además utiliza teoremas sobre rectas paralelas para } \\
\text { la medida de los ángulos representados. }\end{array}$ & \\
& & \\
\hline
\end{tabular}


Continúa Tabla 4

Competencia

Evaluación de evidencias

Nivel en el que se

encuentra

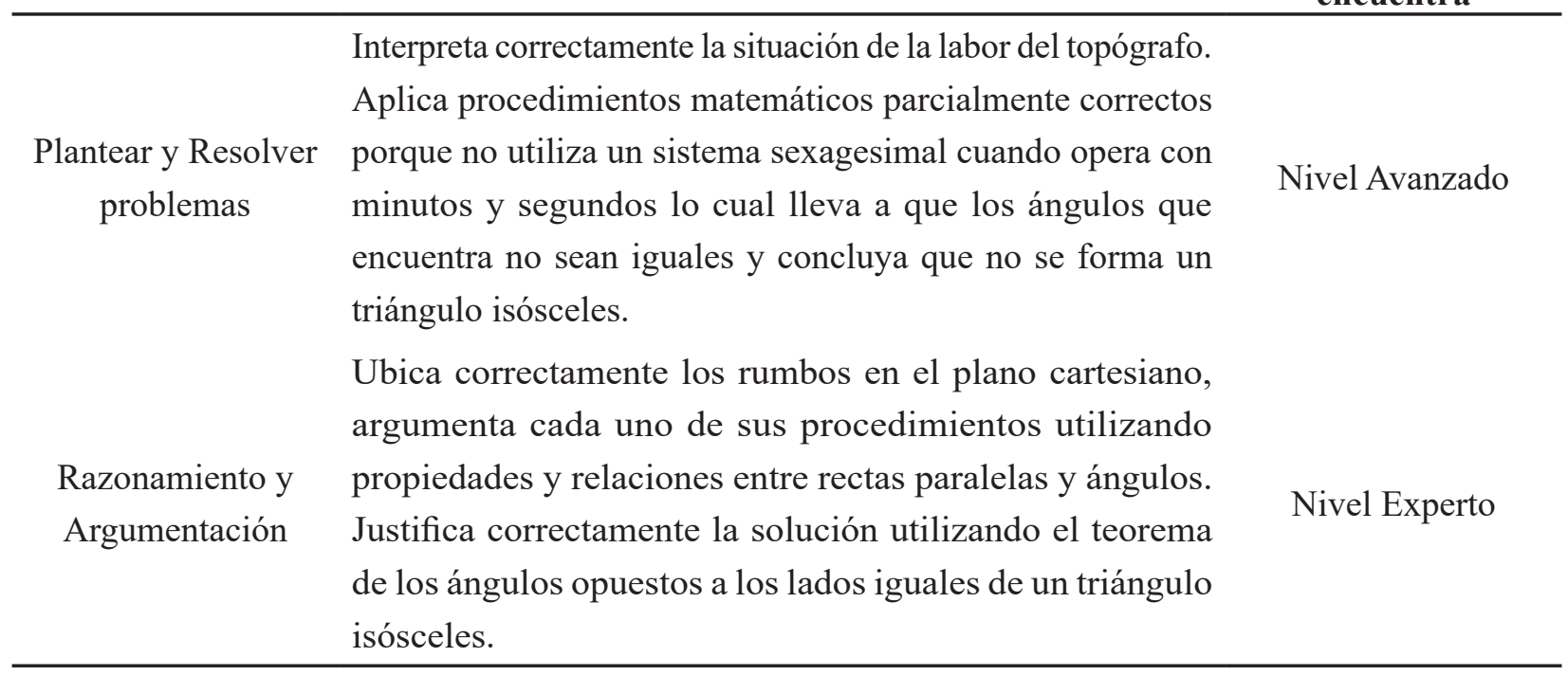

Elemento 5. La Tabla 5 ejemplifica el conteo de frecuencias, según los resultados de desempeño de todos los participantes en el ítem 6. Esto se realizó para todos los ítems.

\section{Tabla 5}

Valoración general para el Ítem 6

\section{Competencia}

\section{Logro}

6 de 15 no comprenden la actividad del topógrafo, no explican sus ideas matemáticas, sus cálculos ni resultados, ubicándose en el nivel nulo.

Comunicación 6 de 15 comprenden la actividad del topógrafo, expresan algunas relaciones entre las direcciones dadas con otros conceptos matemáticos, pero no reflexionan sobre sus ideas matemáticas, ubicándose en el nivel inferior.

1 de 15 alcanza el nivel experto.

4 de 15 no descodifican la representación de la actividad del topógrafo ni la interpretan de manera correcta, no logran codificarla de acuerdo a sus experiencias, ubicándose en el nivel nulo.

Representación

8 de 15 descodifica la actividad del topógrafo, pero no la interpretan correctamente y al codificarla en un cambio de representación lo hacen de forma incorrecta, se ubican en un nivel inferior.

1 de 15 logra ubicarse en el nivel de experto. 


\section{Continúa Tabla 5}

\section{Competencia}

\section{Logro}

6 de 15 no resuelve el problema aplicando procedimientos matemáticos ni propiedades entre conceptos, ubicándose en el nivel nulo.

Plantear y

Resolver

problemas

4 de 15 soluciona el problema interpretando la actividad del topógrafo y establece relaciones entre las direcciones dadas con otros conceptos matemáticos, pero, son deficientes o su procedimiento matemático es parcialmente correcto, se ubican en el nivel inferior al esperado.

1 de 15 interpreta correctamente la situación, pero aplica procedimientos matemáticos parcialmente correctos porque no utiliza el sistema solicitado, se ubica en nivel avanzado.

5 de 15 no conoce ni sigue un razonamiento matemático en sus ideas matemáticas, no justifica sus cálculos ni resultados ubicándose en el nivel nulo.

Razonamiento

y

Argumentación
8 de 15 conoce y sigue el razonamiento matemático de las direcciones como lados de la figura, pero su justificación de resolución es incompleta o incorrecta, ubicándose en el nivel inferior.

1 de 15 se ubica en el nivel inicial para la reflexión y 1 de 15 logra estar en el nivel experto.

\section{Resultados}

Realizado el procedimiento mostrado en el análisis de los 12 ítems, se presenta el resumen de resultados alcanzados en la prueba de desempeño, según los niveles de logro en cada una de las cuatro competencias estudiadas.

\section{Competencia Comunicación}

Como se puede observar el nivel de desarrollo en la competencia comunicación (Figura 5), en los ítems cuyo nivel de complejidad corresponde a reproducción de conceptos y procedimientos matemáticos, se observa que hay un desempeño destacado que ubica a la mayoría de los estudiantes a nivel de expertos (categoría de mejor desempeño). En la categoría nulo se ubican los casos que no presentaron evidencias para poder evaluar el dominio de la competencia y en la categoría inferior, los casos cuyos rasgos muestran un desempeño incoherente con la tarea solicitada. 


\section{Figura 5}

Nivel de desarrollo de competencia comunicación

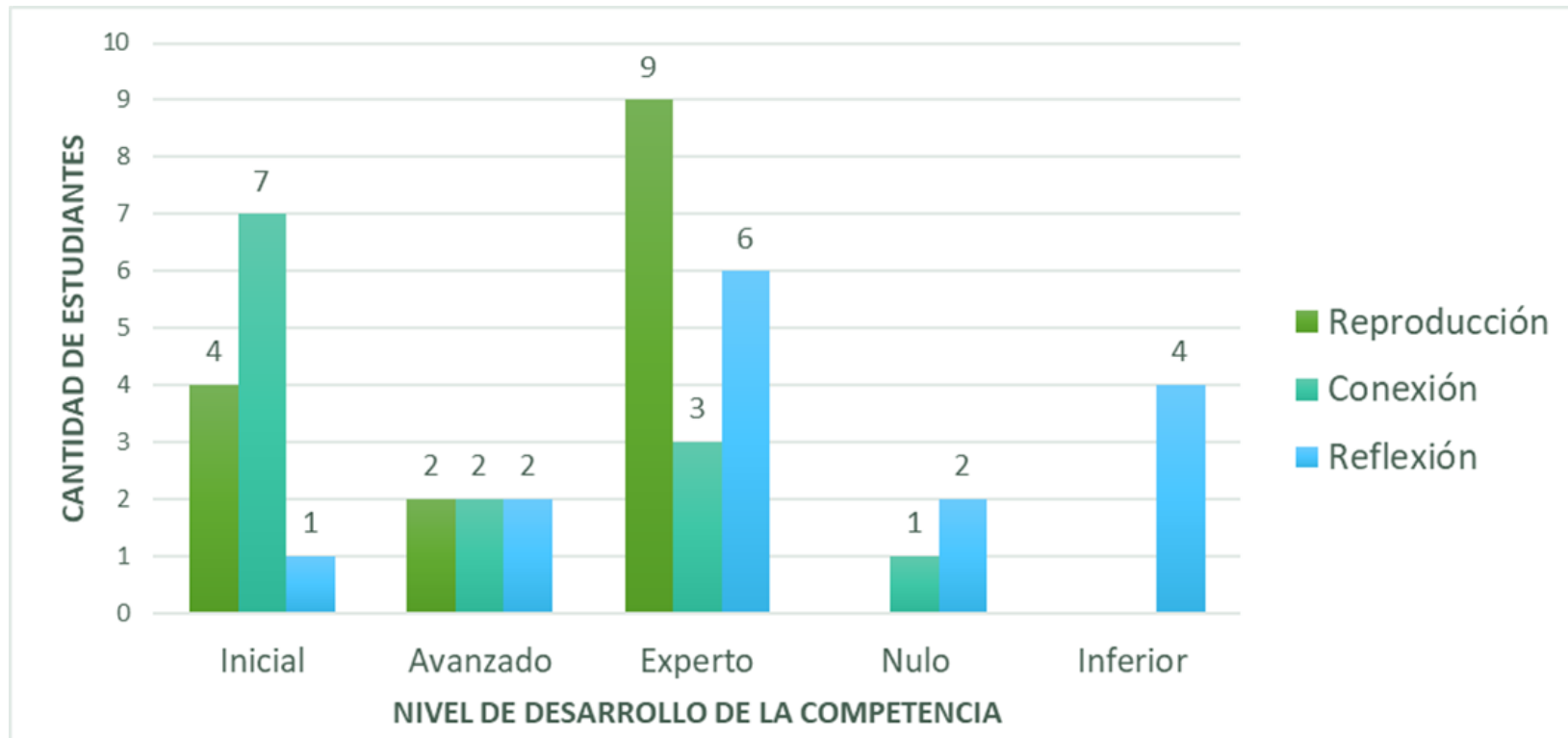

Nota. Fuente: Elaboración de los autores.

En el desarrollo de los ítems con un nivel de complejidad de conexión, donde el estudiante debe conectar saberes de otras ramas del conocimiento o de la misma matemática, los estudiantes alcanzan, en su mayoría, la categoría inicial. Para los ítems que requieren mayor demanda cognitiva, y además de establecer conexiones requieren reflexión (pensamiento autónomo y creatividad), se ubican seis estudiantes en el nivel experto, pero de igual manera cuatro casos se ubican en un nivel inferior al propuesto en el diseño de los ítems.

\section{Competencia Representación}

En la competencia representación, de nuevo se puede observar en la Figura 6 que la mayoría de estudiantes alcanzan resolver los ítems cuyo nivel de complejidad corresponde a reproducción, pero también hay más estudiantes que resuelven los ítems que corresponden a un nivel de complejidad de conexión, la mayoría de los participantes están en la categoría de expertos. Para el nivel de complejidad de reflexión, una buena representación del grupo se ubica en la categoría de avanzados. También se observa que hay más estudiantes cuyos rasgos muestran un desempeño incoherente con la tarea solicitada. 


\section{Figura 6}

Nivel de desarrollo de competencia representación

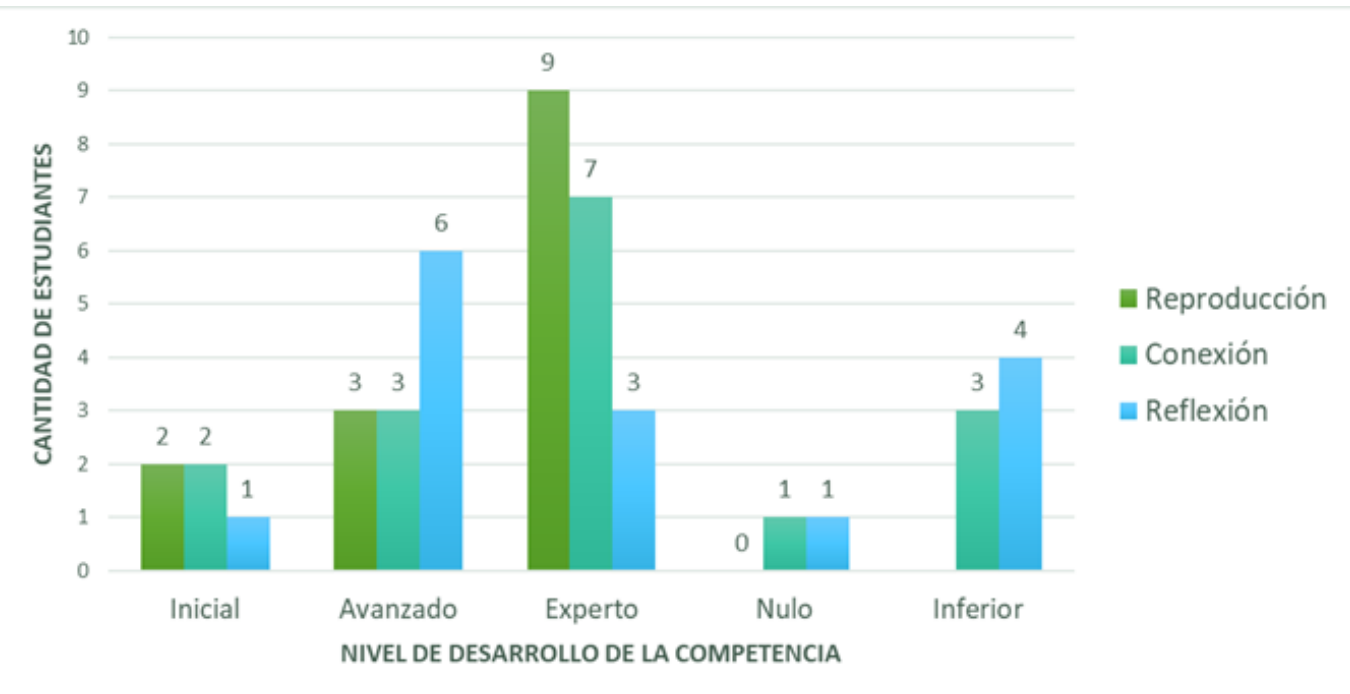

Nota. Fuente: Elaboración de los autores.

\section{Competencia Plantear y Resolver Problemas}

En cuanto a la competencia plantear y resolver problemas (Figura 7), más estudiantes responden mejor a tareas con un nivel de complejidad de reproducción (categoría expertos). Es la competencia donde más estudiantes se registran en la categoría de expertos, incluyendo el desarrollo de tareas con nivel de complejidad tanto de conexión como de reflexión. Se mantiene el patrón de estudiantes cuyos rasgos muestran un desempeño incoherente con la tarea solicitada.

\section{Figura 7}

Nivel de desarrollo de competencia plantear y resolver problemas

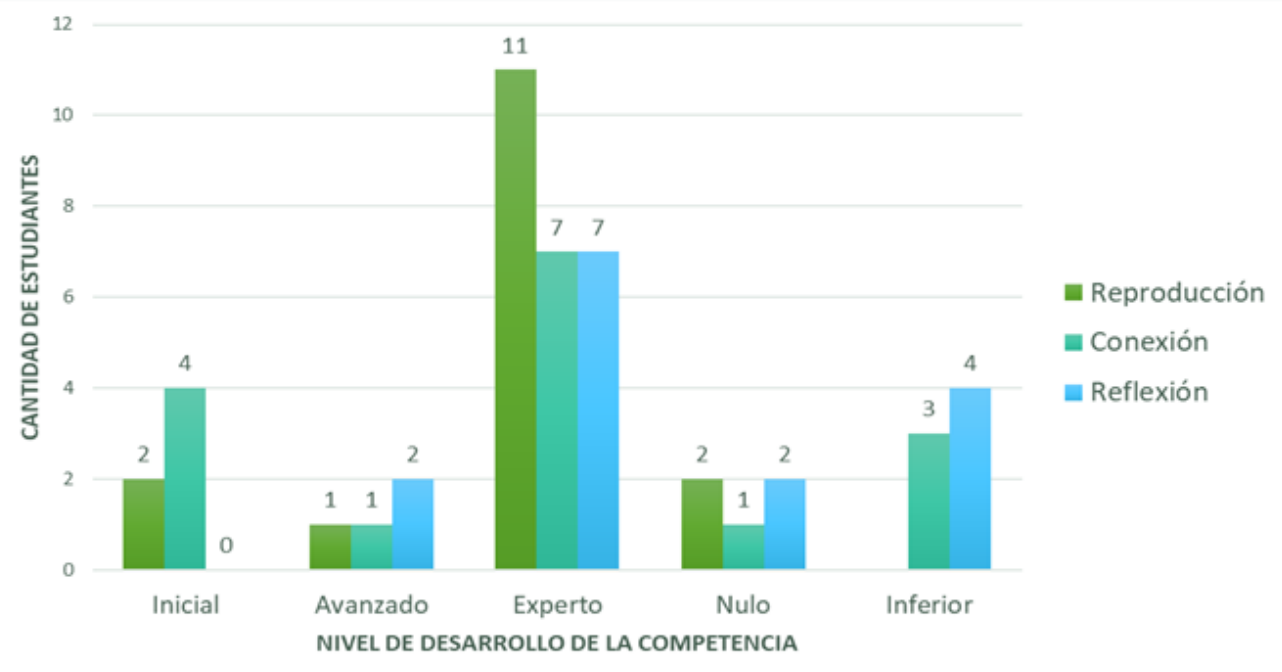

Nota. Fuente: Elaboración de los autores. 


\section{Competencia de Razonamiento y Argumentación}

Como se puede observar el nivel de desarrollo en la competencia de razonamiento y argumentación (Figura 8), de nuevo el mejor desempeño se da en las tareas cuya demanda cognitiva es la más baja, la de reproducción de conceptos y procedimientos matemáticos. Sin embargo, es la competencia donde más estudiantes alcanzan la categoría de expertos tanto a nivel de complejidad de conexiones como de reflexión. De igual manera para tareas con nivel de complejidad de conexiones más estudiantes tienen desempeños incoherentes.

\section{Figura 8}

Nivel de desarrollo de competencia de razonamiento y argumentación

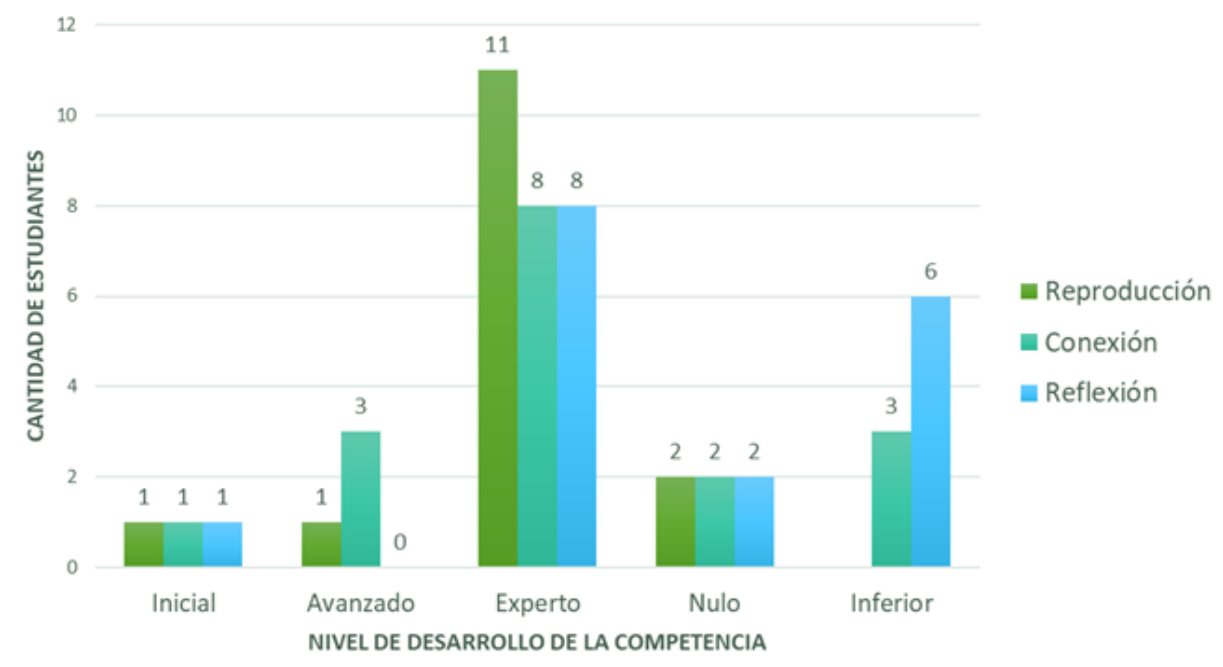

Nota. Fuente: Elaboración de los autores.

\section{Discusión de Resultados}

El propósito del estudio es describir el nivel de desarrollo que muestran los estudiantes del segundo año de la carrera de Matemáticas de la UPNFM de la modalidad presencial, en las competencias matemáticas seleccionadas. En este sentido, se hace una breve descripción de los resultados por cada una de las competencias.

\section{De la Competencia de Comunicación}

El hallazgo recurrente en el estudio sobre esta competencia es que los estudiantes se muestran expertos para comprender y saber expresarse en la reproducción o reiteración de conocimientos ya practicados de tareas o situaciones relacionadas con estimación de magnitudes, aplicaciones de la derivada, enunciados traducidos a expresiones algebraicas y aplicaciones del Teorema de Pitágoras, mostrando dominio en la capacidad de expresarse, tanto oral como por escrito en 
asuntos con contenidos matemático (Niss, 2002). Los estudiantes se dan a entender, justificando el procedimiento utilizado que los lleva a resolver una situación problemática de esta índole. A nivel de reproducción, los estudiantes utilizaron distintas formas de lenguaje (escrito argumentativo, icónico y gráfico) para mostrar el desarrollo de sus ideas matemáticas, comprender el problema y así llegar a una solución.

Cuando se trata de comunicar en el nivel de complejidad de conexión la mayoría se volvió aprensiva, comunican a medias o simplemente no lo hacen, lo que dificultó la evaluación de la competencia. Al momento de explicar asuntos que implican relaciones, enlaces o conexiones de diferentes aspectos para alcanzar una solución de tareas o situaciones sobre los temas de representación de objetos en el espacio, aplicaciones de ecuaciones lineales en una variable y lectura, interpretación y representación gráfica de una función lineal de los contenidos de las áreas de geometría y algebra, según los resultados, la mayoría de los estudiantes demuestran, que están por debajo de esa demanda cognitiva, es decir, no dieron las evidencias pedidas que demostraran poseer dominio de esta competencia.

Para Goñi (2008) la competencia matemática de comunicación es un factor importante para potenciar las matemáticas y la resolución de problemas, ya que, no es posible desarrollar un proceso de pensamiento largo y complejo sin contar con elementos comunicativos y expresivos. Esto se evidencia particularmente en el ítem \#6 donde las pocas evidencias permiten asumir que la situación y el contexto no fueron comprendidos y por tanto no hubo muestras de comunicación que permitiera evaluar la competencia. En general, se encontró dificultades por parte del estudiante al momento de explicar asuntos que implican cálculos o resultados de relaciones complejas entre ellas relaciones lógicas de tareas sobre temas de aplicaciones de direcciones en $\mathrm{R}^{2}$, ángulos alternos internos de rectas paralelas y representación de objetos en el plano de los contenidos de geometría y algebra, incluso llegan a niveles anteriores del propuesto.

Esta experiencia permitió contextualizar las matemáticas como un área de conocimiento en el que el elemento comunicativo ocupa un lugar central, sin embargo, se requiere incorporar como manifiesta Monaghan (2006) actividades que impliquen la escritura de datos, graficas en sus distintas formas de representación, las operaciones que realiza. Todo ello hace uso de formas expresivas que ayudan a organizar el proceso de pensamiento necesario para la resolución del problema, en un tipo de lenguaje usado y necesario para desarrollar una fluidez matemática. 


\section{De la Competencia de Representación}

A un nivel de complejidad de reproducción, los resultados del desempeño muestran que los estudiantes han logrado el nivel experto. Muestran competencias en los contenidos sobre geometría, álgebra y en tareas donde el estudiante se encuentra en situaciones familiares y dichos problemas son reiterativos. En cambio, en los ítems que evalúan la competencia en un nivel de complejidad de conexión, estos resultados muestran que los estudiantes son capaces de construir patrones geométricos, pero presentan una debilidad al analizar datos estadísticos y tener que descodificar una representación gráfica o una interpretación de dichos datos y viceversa. La capacidad de establecer conexiones son deficientes en el área de estadística lo que provoca que sus cambios de representación sean parciales e incompletas.

En el nivel de complejidad de reflexión, los resultados muestran grandes deficiencias en particular en el ítem seis donde el nivel de logro de la competencia corresponde a un nivel de inicial. Esto se evidenció al no poder representar los rumbos del topógrafo correctamente y al no reflexionar que debían formar un triángulo. Se muestra que no hay indicios de desarrollo de la competencia de representación (se ubica en un nivel inferior) cuando se evalúa niveles de complejidad más altos y de contenidos o áreas más complejas.

\section{De la Competencia Plantear y Resolver Problemas}

La capacidad de reproducción tiene una marcada presencia en esta competencia, los estudiantes muestran un logro experto. En el nivel de reproducción, quiere decir que exponen y formulan problemas reconociendo y reproduciendo tareas ya practicadas de los temas de estimación de magnitudes, aplicaciones de la derivada, enunciados traducidos a expresiones algebraicas y aplicaciones del Teorema de Pitágoras. Traducen una situación problemática de un contexto real al mundo matemático, resuelven problemas a partir de sus vivencias (Segarra, 2004). Sobre este hallazgo el factor determinante en este tipo de actividades es que los estudiantes ya contaban con elementos conceptuales adquiridos previamente, componente que contribuyó positivamente en el trabajo que desarrollaron.

En esta competencia la mayoría de los estudiantes logró llegar al nivel más alto (experto), establecieron conexiones entre distintas áreas de las matemáticas, lo que llama Perrenoud (2007) movilizar un conjunto de recursos cognitivos (saberes e información) de tareas o situaciones plateadas. Los estudiantes utilizaron algunas de las variables que según Rogoff (1998) influyen en los resultados de la resolución de problemas: la disponibilidad de conceptos en la estructura cognoscitiva, la sensibilidad al mismo, la curiosidad intelectual y la tolerancia a la frustración. 
En lo que conlleva al nivel de reflexión, la mitad del grupo alcanza el nivel experto, arraigando las matemáticas al lugar y contexto social, lingüístico y cultural (Alsina, 1998) representando el uso funcional del conocimiento matemático de tareas sobre temas de aplicaciones de direcciones en $\mathrm{R}^{2}$, ángulos alternos internos de rectas paralelas y representación de objetos en el plano de los contenidos de geometría y álgebra, movilizan además de saberes e información, su capacidad de superar obstáculos para generar preguntas científicas y significativas que les ayuden a encontrar una solución. Evidencia además el uso de un medio que refuerza el aprendizaje del resto de los contenidos curriculares del plan de estudios y la aplicación de las matemáticas a casos prácticos. El resto se distribuye en los niveles de dominio más bajos, es destacable el hecho de que estas actividades solo están asignadas a un bloque de contenidos de la malla curricular y requieren comprensión reflexiva para su realización, existe la posibilidad de no ser acertada la asignación, como sucede en el ítem \# 6 "El topógrafo" en el que solo dos de los 15 participantes se acercó de manera significativa a la solución del problema y que se considera un caso especial ya que marca una diferencia muy pequeña en el nivel de logro general de los estudiantes.

\section{De la Competencia de Razonamiento y Argumentación}

Los resultados de la prueba muestran que los estudiantes en la competencia de razonamiento y argumentación han logrado dominio a nivel de reproducción, son capaces de argumentar sus procedimientos de solución solamente cuando se encuentran en una tarea familiar que implique procesos conocidos de resolución. Pero cuando se encuentran en tareas no familiares que impliquen generalizar una solución son incapaces de justificar su procedimiento de solución. Se puede observar que, en los ítems con niveles de complejidad de conexión y reflexión, los estudiantes no presentan o exponen sus razonamientos a pesar de resolver correctamente dichas tareas. Esto muestra limitaciones comunicativas para explicar sus procesos, se limitan a resolver los problemas, pero no comprueban la solución y en ocasiones no explican los conceptos o propiedades matemáticas que justifican sus razonamientos.

\section{Desde una Mirada General}

Los resultados muestran que en su mayoría los estudiantes alcanzan la categoría de experto en las tareas cuya solución solo requiere la reproducción de conceptos y procedimientos matemáticos, es decir en la reproducción de saberes. En situaciones o tareas más complejas, que requieren hacer conexión de conceptos o de situaciones, o de reflexión, donde se requiere pensamiento autónomo, se observa un menor rendimiento. 
También se observa que más estudiantes responden mejor en tareas de menor nivel de complejidad, (reproducción) relacionadas con las competencias plantear y resolver problemas y razonamiento y argumentación. Esto es coherente con las tradiciones docentes en matemáticas y las competencias que promueve el curriculum de la carrera.

Es importante notar que entre seis y siete de los estudiantes de los 15 que participaron muestran niveles de desarrollo en la categoría de experto (el máximo esperado), aún en situaciones de mayor complejidad de las tareas planteadas, tal como la necesidad de establecer conexiones ante problemas no rutinarios que requerían conectar conceptos de otras áreas y poder representar de distintas formas una misma situación, o de reflexión, ante situaciones que requería movilizar competencias que requieren cierta comprensión y reflexión por parte del alumno, creatividad para identificar conceptos o enlazar conocimientos de distintas procedencias.

El nivel de desempeño es bajo en la competencia comunicación y todavía más bajo, en competencia de representación. Estas son competencias, que posiblemente todavía no son objeto de suficiente atención en la gestión de los aprendizajes y posiblemente no se incluyen de manera intencional en la planificación didáctica de las trayectorias formativas de los estudiantes del Profesorado en Matemáticas de la UPNFM. Caso contrario a la competencia de plantear y resolver problemas y razonamiento y argumentación, los estudiantes cuentan con mejor experiencia dada la tradición de la carrera del Profesorado en Matemática, estas competencias son elementos transversales en el pénsum de la carrera lo que contribuyó positivamente en el trabajo que desarrollaron los participantes en esta investigación.

Entre las limitaciones que encontramos fue al momento de estudiar la competencia razonamiento y argumentación, a la luz de los datos se considera que para futuras investigaciones es necesario indagar más en profundidad y considerar todas las competencias matemáticas. También, parece interesante que una futura investigación está en profundizar en los contenidos de aplicación de vectores en $\mathrm{R}^{2}$, que fue el contenido que presentó resultados más bajos en comparación con los demás.

Para finalizar conviene señalar que el plan de estudios de la carrera del profesorado en matemáticas de la UPNFM, no especifica los niveles de competencia a lograr en cada año de carrera o por ciclos formativos, se considera que un esfuerzo sistemático integral y continuo del departamento de matemática puede lograr fortalecer el desarrollo de las competencias hasta llevar a más estudiantes a fortalecer tales competencias, para ello es importante precisar estos aspectos en el diseño curricular. 
Punto clave en ese proceso, es enfatizar la importancia sobre el desarrollo de las competencias matemáticas en los futuros docentes de matemáticas, esto se logrará en la medida que el proceso educativo sea planificado con base a las cuatro variables que hemos considerado: contenido, contexto, nivel de complejidad y situación o tarea. Para lograrlo, es necesario institucionalizar conocimientos y experiencias sobre las competencias matemáticas, cómo se gestionan, precisar los indicadores de logro de tales competencias y cómo gestionar y evaluar los resultados de aprendizaje.

\section{Comentarios Finales}

Conviene señalar la posición estratégica de la carrera que se expresa:

Tomando en cuenta los factores determinantes en el proceso de enseñanza aprendizaje de la matemática como ser el análisis epistemológico de las matemáticas y su relación con las tendencias actuales en educación matemática donde el alumno, el profesor y el contexto juegan un papel fundamental. La carrera de Profesorado en Matemáticas en el Grado de Licenciatura se constituye en un sistema de formación docente en matemáticas para el Sistema Educativo. Desde esta perspectiva los actores del proceso (educandos y profesores) deben integrar y apropiarse de los conocimientos de la educación matemática misma y de sus tendencias actuales. Y así proporcionar el fundamento teórico para nuevos procesos educativos a nivel de postgrado. Dicho sistema tomará en consideración tres ejes transversales los cuales son: el uso de la tecnología en el aprendizaje de la matemática, la resolución de problemas como fin del aprendizaje y como medio de enseñanza y el desarrollo del pensamiento matemático (UPNFM, 2008, p.15).

En consonancia con el actual modelo de la UPNFM, los diseños basados en competencias y resultados de aprendizaje, requiere establecer metas y criterios para mejorar la educación matemática y científica, fomentar las reformas curriculares, impulsar la mejora de la enseñanza y el aprendizaje y promover la investigación de la práctica misma del docente. El monitoreo que permita conocer los aprendizajes alcanzados por sus estudiantes, las dificultades que obstaculizan esos logros y proponer estrategias para la mejora son pieza clave para alcanzar la posición estratégica de la carrera de matemáticas.

Esta investigación es un aporte metodológico hacia esa posición a través de la operacionalización de competencias matemáticas para orientar su evaluación hacia indicadores significativos de logro de aprendizajes, lo cual debe estar en la base de la planificación y la evaluación de los aprendizajes de la matemática. 


\section{Reconocimientos}

La investigación Nivel de desarrollo de las competencias matemáticas en estudiantes de segundo año de la carrera del profesorado en matemáticas del año 2013 ha sido desarrollada gracias a la colaboración del Fondo de Apoyo a la Investigación de la Universidad Pedagógica Nacional Francisco Morazán de Honduras. Agradecemos a todos los compañeros, docentes, estudiantes, colegas y demás personal que ayudaron a concluir de manera exitosa esta investigación.

\section{Referencias}

Abrantes, P. (2001). Mathematical competence for all: Options, implications and obstacles, Educational Studies in Mathematics, 47, 125-143.

Alsina, A. (2004). Desarrollo de competencias matemáticas con recursos lúdico-manipulativos. Madrid, España: Narcea.

Barrales, V. L (2012). El enfoque educativo basado en competencias, un reto que enfrenta la Universidad Veracruzana. Educación, XXI(41), 23-39.

Beneitone, P. Esquetine, c., Gonzáles, J., Marty, M., SiufyM. y Wagenaar, R. (2007) (Eds). Reflexiones y perspectivas de la Educación Superior en América Latina, en el Informe final Proyecto Tuning-América Latina. 2004-2007. Deusto: Universidad de Deusto.

Blanco, A. (2009). Desarrollo y evaluación de competencias en educación superior. Madrid: Editorial Narcea.

CMEC (2011). Report on the Pan-Canadian Assessment of Mathematics, Science, and Reading 2010.

CSUCA (2013). Marco de Cualificaciones para la Educación Superior Centroamericana. Resultados de aprendizaje para Licenciatura, maestría y doctorado. Una aproximación desde la visión académica. Consejo Superior Universitario Centroamericano (CSUCA)-Alfa. Unión Europea.

D’Amore, B. (2008). Bases filosóficas, pedagógicas, epistemológicas y conceptuales de la Didáctica de la Matemática. México: Reverté S.A.

Delors, J., Al Mufti, I., Amagi, I., Cameiro, R., Chung, F.,Geremek, B., Gorham,W., Kornhauser, A., Manley, M., Padrón, M.,Savané, M., Singh, K., Stavenhagen,R., Won, M. y Nanzhao, Z. (1996). La educación encierra un tesoro. Informe a la UNESCO de la Comisión internacional sobre la educación para el siglo XXI, Madrid, España: Santillana/UNESCO. pp. 91-103 
González, V. y González, R.M. (2008) Competencias genéricas y formación profesional: Un análisis desde la docencia universitaria. Revista Iberoamericana de Educación, N.47, 185-209.

Goñi, J. M. (2008). El desarrollo de la competencia matemática. Barcelona, España: Grao.

Green, I., López, L., Chambasis, R., Valladares, A., Díaz, M., Martínez, L. y Molina, D. (2014). Aproximación a la operacionalización de competencias matemáticas: Una estrategia necesaria para la práctica curricular. Paradigma Estudiantil, 1(1), 10-19.

ICFES. (2012). Pruebas Saber 3o., 5o. y 9o. Lineamientos para las aplicaciones muestral y censal 2012. Bogotá: ICFES.

Ministerio de Educación Nacional (1998). Matemáticas. Lineamientos curriculares. MEN.Bogotá.

Ministerio de Educación Nacional. (2003) Estándares Básicos en Competencias Matemáticas. Colombia

Monaghan, F. (2006). Thinking aloud together, Mathematics Teaching, 198, 12-15

Mullis, I., Martin, M., Ruddock, G., O’Sullivan, C. y Preuschoff, C. (2012). Timss 2011 Assesment Frameworks. TIMSS y PIRLS International Study Center and International Association for the Evaluation of Educational Achievement.

NCTM (2000). Principios y Estándares para la Educación Matemática. España: Sociedad Andaluza de Educación Matemática Thales.

Niss, M. (2002). Mathematical competencies and the learning of mathematics: the Danish KOM project. Dinamarca: IMFUFA, Rokilde University.

Niss, M. y Hojgaard, T. (2011). Competencies and Mathematical Learning Ideas and inspiration for the development of mathematics teaching and learning in Denmark. English Edition. Publicaciones IMFUFA, Roskilde University, Denmark.

OCDE (2006). PISA marco de la evaluación. Conocimientos y habilidades en Ciencias, Matemáticas y Lectura. España: Santillana.

Perrenoud, P. (2007). Diez nuevas competencias para enseñar. Cuarta edición. Barcelona, Graó.

Rogoff, B. (1993). Aprendices del pensamiento. El desarrollo cognitivo en el contexto social. Buenos Aires, Argentina: Paidós. 
Segarra, Ll. (2004). Problemates. Colección de problemas matemáticos para todas las edades. Barcelona, España: Graó.

Tobón, S. (2008). La formación basada en competencias en la educación superior: El enfoque complejo. México: Universidad Autónoma de Guadalajara,

Tobón, S. (2010). Secuencias didácticas: aprendizaje y evaluación de competencias.

UPNFM (2008). Plan de Estudio de la Carrera de Matemáticas en el Grado de Licenciatura. Tegucigalpa, Honduras. Universidad Pedagógica Nacional Francisco Morazán 
Ivy Lou Green Arrechavala, Rosa Janeth Chambasis Cruz, Ledher Manuel López Urquía

118 <Paradigma> Revista de Investigación Educativa | Año 27 | №45 | Junio-Diciembre 2021 | ISSN: 1817-4221 | EISSN: 2664-5033

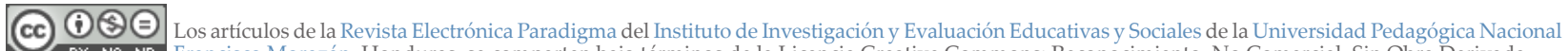

EY No ND Francisco Morazán, Honduras, se comparten bajo términos de la Licencia Creative Commons: Reconocimiento, No Comercial, Sin Obra Derivada 